\title{
How Anti-Humeans Can Embrace a Thermodynamic Reduction of Time's Causal Arrow
}

\author{
Eli I. Lichtenstein
}

\begin{abstract}
Some argue that time's causal arrow is grounded in an underlying thermodynamic asymmetry. Often, this is tied to Humean skepticism that causes produce their effects, in any robust sense of 'produce'. Conversely, those who advocate stronger notions of natural necessity often reject thermodynamic reductions of time's causal arrow. Against these traditional pairings, I argue that 'reduction-plus-production' is coherent. Reductionists looking to invoke robust production can insist that there are metaphysical constraints on the signs of objects' velocities in any state, given other-including far later-states' properties. The Past Hypothesis may thus be a metaphysical condition, not a physical law.
\end{abstract}

\section{Introduction}

Broadly inspired by Boltzmann (1895), several commentators have argued that time's causal arrow, or the asymmetry whereby causes typically precede their effects (Price and Weslake 2009), is grounded in an underlying thermodynamic asymmetry. This underlying thermodynamic arrow of time may be characterized in terms of the entropy gradient of a large-scale or all-encompassing physical system (Dowe 1992; cf. Earman 1974, Horwich 1987). Or it may be characterized in terms of more basic statistical mechanical phenomena-e.g., by commentators who suggest that the thermodynamic arrow of time is implied by the conjunction of (i) the Past Hypothesis (that the entropy of the universe's initial macrostate was very low), (ii) the equiprobability of all microstates that realize this initial macrostate, (iii) the present macrostate, and (iv) the dynamical laws governing microstates (Horwich 1987; Albert

\footnotetext{
$\dagger$ Forthcoming in Philosophy of Science (doi: 10.1086/715514)

$\$$ Earlier versions of this paper were presented in 2019 at an IAPT meeting in Boulder and SMS meeting in Toronto - thanks to organizers and audiences at both. For helpful feedback, I am especially grateful to David Albert, Gordon Belot, Harjit Bhogal, Craig Callender, John Carroll, Nina Emery, Alison Fernandes, Tyler Hildebrand, Ezra Rubenstein, and Michael Tooley.
} 
2000, 2015; Loewer 2012a). Regardless, the basic program of thermodynamic reduction, or grounding time's causal arrow in an underlying thermodynamic arrow, is often undertaken in a spirit of broadly Humean skepticism that causes produce their effects, at least in any very robust sense. ${ }^{1}$ For instance, while Reichenbach allows that "[t]he cause produces the effect" and "the effect records the cause," he qualifies that "the word 'produces' is a statistical concept"-so the merely "emotive" terms 'produce' and 'record' "find an explication in the statistical definition of time direction" (Reichenbach 1956, 156).

Conversely, commentators friendly to stronger notions of natural necessity often take their anti-Humeanism to stand in tension with thermodynamic reduction. For example, Maudlin claims that the fundamental laws of nature are "ontologically primitive" $(2007,12-15)$ and that the "direction of the passage of time" is "not to be reduced to, or analyzed in terms of, anything else," including the entropy gradient of the universe (2007, 118 and 142). For Maudlin, these claims are deeply intertwined. Laws of nature "operate to generate or produce" all later states of the universe from its initial state, and the direction of time is the direction in which the laws exercise this productive power (Maudlin 2007, 174). So if either the laws or the direction of time "ontologically depend[ed]" on the "global structure" of the Humean Mosaic, Maudlin believes, the laws' productive power vis-à-vis later states of the universe would be compromised $(2007,175)$.

Below, I challenge this traditional pairing of positions. I generalize Maudlin's notion of law-governed 'production', and argue that the resultant generalized notion better illuminates the basic tension between thermodynamic reductionist accounts of time's causal arrow and anti-Humean accounts of natural necessity. I suggest that thermodynamic reductionists who wish to appeal to robust notions of 'production' should insist that there are metaphysical constraints on what the signs of objects' velocities in a given state can be, given other-including later-states' properties. Hence, one could claim that not all physically possible states are metaphysically possible initial states. I conclude that there is no deep tension in a scheme of reduction-plus-production.

1 Note that 'thermodynamic reduction' is not always used in this specific sense, in the contemporary literature. 


\section{Maudlin's Anti-Reductionism and Generalized Metaphysics of 'Production'}

Maudlin does not clearly elaborate the connection between his antireductionism about laws of nature and his anti-reductionism about the direction of time, but his basic intuition is plausibly as follows. The direction of time is, qua 'productive' or causal arrow, ${ }^{2}$ the direction in which the laws exercise their 'productive' force. ${ }^{3}$ So it would apparently involve a damaging kind of circularity if the laws of nature or the ('productive') direction of time were to ontologically depend upon later states of the universe-i.e. upon the outputs of productive processes that presuppose the laws and the direction of time. Yet thermodynamic reductionists evidently claim just this. For thermodynamic reductionists in the present sense, that is, the direction of time in which laws produce output states - or the direction of time in which causes produce their effects-is grounded in an underlying thermodynamic asymmetry. And this thermodynamic asymmetry is one between the thermodynamic properties of states at different times, including the (later) states that Maudlin takes to be 'produced' by laws of nature.

To elaborate this basic worry in a way that is less closely bound to Maudlin's framework, but correspondingly more relevant for my broader purposes here, we may consider the following problem. If a temporally-extended portion of the Humean Mosaic must exist in order for the direction of time to be well-defined, then it seems to follow that a temporally-extended portion of the Mosaic must exist in order for the signs of objects' velocities or momenta in a given state of the universe to be determinate. Imagine, for example, a state $S$ in which a baseball traveling at $90 \mathrm{mph}$ is halfway between a hand and a window, surrounded in time in one direction by a state in which the baseball is touching the hand and, in the other direction, by a state in which the baseball is touching the window. Is the baseball in $S$ moving $90 \mathrm{mph}$ away from the hand and towards the window, or $90 \mathrm{mph}$ away from the window and towards the hand?

It seems that if thermodynamic reduction of time's causal arrow is correct, and if one is a robust realist about the direction of the passage of time, then the answer to this question will depend on how the baseball-at-hand and baseball-at-window events

2 Maudlin $(2007,176)$ suggests that "the relevant notion of causation is a form or aspect of production."

3 Maudlin $(2007,174-175)$ claims that "[t]he basic temporal asymmetry of past-to-future underlies the very notion of production itself." 
are situated with respect to a broader physical system - e.g. a global entropy gradient, like that between a low-entropy 'Big Bang' in the past and a hypothetical maximumentropy 'heat death' of the universe in the future. Crucially, the issue here is ontological, not epistemological: the problem is not just that an observer cannot know which way the ball is moving in $S$ without having any information about other states, but rather that it is genuinely indeterminate which way the ball is moving in $S$, except in the context of other states. Thermodynamic reduction thus seems to entail that the dynamical properties of any given state of the universe, saliently including objects' velocities or momenta therein, are not entirely well-defined unless other states also exist. And this stands in tension with Maudlin's attribution of a kind of ontological independence to the universe's initial state.

Depending on what one thinks it takes for a state to 'exist', a thermodynamic reductionist sympathetic to Maudlin's account of law-governed production might infer either that the universe's initial state depends for its existence on later states, or else simply that the signs of objects' initial velocities depend for their existence on later states. Either way, there is a problem here for the thermodynamic reductionist who wishes to adopt Maudlin's account of law-governed production: thermodynamic reduction apparently entails that information from later states of the universe is required to fix the signs of objects' velocities in earlier states, including the initial state. In this sense, the laws' activity at the beginning of time is apparently sensitive to information from later times. But information flowing backwards in time sounds spooky. And it runs counter to Maudlin's implicit claim that the laws' initial productive activity is temporally local, i.e., fully determined and made possible by things proximate in time to the initial state's obtaining. Let us call this the Direction of Motion Problem.

One might object that this 'problem' is not a real problem. Is it really true, for instance, that thermodynamic reduction 'entails that information from later states of the universe is required to fix the signs of objects' velocities in earlier states,' as I claimed above? After all, if time's direction is reduced to the Past Hypothesis and a probability measure, then it is not at all clear that this involves any states later than the initial state. By way of response, I agree that if one stipulates a low-entropy initial condition (or state shortly after the Big Bang, etc.), assumes equiprobability of microstates realizing this macrostate, and posits normal kinds of physical laws, then 
this is plausibly enough to guarantee that entropy is overwhelmingly likely to increase after this low-entropy state-assuming that law-governed processes governing microstate-evolution are allowed to 'run' forward in time. But this simply begs the question that I am interested in, on behalf of reductionists of the sort I am talking about: how do we know that the low-entropy state we stipulate as a boundary condition is in the past, rather than the future? If we just stipulate that this low-entropy boundary condition is in the past, then we have already stipulated a direction of time between it and us (in the present). To see this more clearly, imagine that the universe contains only a gas in a box; that at one end of time the gas molecules are spread out evenly in the box; and that at the other end of time the gas molecules are clumped together in one corner of the box. Did this gas spread out? Or did it start spread out, and then clump up? A thermodynamic reductionist in the present sense must, as such, be committed to appealing to something like a holistic entropy gradient to settle this question-hence, also, to determine the sign of the gas molecules' velocities ('spreading out or clumping up?'). For true thermodynamic reductionists, it is precisely the low entropy of the Past Hypothesis that fixes it as the past boundary condition.

Note that what is mainly at issue, in the Direction of Motion Problem, is not Maudlin's anti-reductionist account of law-governed production. Rather, it is his commitment to what we might call a generic metaphysics of production. To see this, imagine a metaphysics in which the loci of productive power are not laws of nature, but rather physical objects. For instance, suppose that the Sun and planets produce the planets' orbits around the Sun, rather than that the laws of nature do so. On this view, too, the same basic worries about thermodynamic reduction arise. Whether we want to say that the laws of nature produce later states of the universe from earlier states, or that the Sun and the planets produce later states of the solar system from its earlier state(s), we will evidently have to be able to say which of two given states is earlier than the other, or at least what the signs of objects' velocities in a given state are. Consequently, those who think that thermodynamic reduction conflicts with one or both of these aspects of determining the universe's initial state, or its direction of motion, should infer that thermodynamic reduction stands in tension with any kind of metaphysics of production. 


\section{Reduction-Plus-Production}

What exactly does a metaphysics of production, in this general sense, involve? Three principles seem essential to any metaphysics of production broadly in line with Maudlin's:

(P1) Things that are produced depend for their existence upon that which produces them.

(P2) If $X$ - the laws of nature, physical particles, fields, etc. - produces state $B$ from state $A$, then nothing produces $A, X$, or the signs of objects' velocities in $A$, from $B$.

(P3) If $X$ produces $Y$ from $Z$, then $X$ and $Z$ do not depend for their existence upon $Y$.

However, a slightly weaker metaphysics of production, involving P1 and P2 but not P3, is consistent with a thermodynamic reduction of time's causal arrow.

The sense in which the direction of motion of objects in the universe's initial state does 'depend for its existence' on later states, according to thermodynamic reductionists, is different from the sense in which the initial direction of motion does $n o t$ 'depend for its existence' on later states, according to a generic anti-Humean metaphysics of production. Here note, first, that if 'producing' is understood to be a temporal process-i.e. if $Y$ can be 'produced' by $X$ only if $X$ exists before $Y$ doesthen the reductionist clearly does $n$ ot think that the universe's initial state is 'produced' by later states or by the global structure of the Humean Mosaic.

The opponent of reduction-plus-production thus confronts a dilemma. On the one hand, if we assume that 'ontological dependence' is (like 'production') a temporal notion in P1-P3 - and if we avoid equivocating - then reductionists should not accept the holistic principle $\mathrm{H}$ :

(H) The direction of motion in the universe's initial state depends for its existence upon the global structure of the Humean Mosaic, or at least upon some later state(s).

On the other hand, if 'ontological dependence' can be atemporal, then reductionists can accept $\mathrm{H}$-but not without thereby sacrificing the intuitive motivation for P3, 
which rests on the assumption that things cannot ontologically depend upon things happening in the future, just as production cannot run backwards in time. In other words, the plausibility of $\mathrm{P} 3$ is underwritten by the implicit premise $\mathrm{P} 3 *$ :

(P3*) If $X$ exists before $Y$ exists, then $X$ does not depend for its existence upon $Y{ }^{4}$

The critic of reduction-plus-production must argue that $\mathrm{P} 1-\mathrm{P} 3$ or $\mathrm{P} 3 *$ is incompatible with $\mathrm{H}$. But, insofar as it is plausible, $\mathrm{H}$ clearly involves an atemporal notion of ontological dependence, distinct from the temporal notion of ontological dependence presupposed by $\mathrm{P} 3$ and $\mathrm{P} 3 *$.

\section{An Analogy Between Thermodynamic Reduction and Best-Systems Accounts of Laws}

Even if one disapproves of positing a radically new form of ontological dependence, there are familiar examples of ontological dependence that more closely resemble what a thermodynamic reductionist who advocates anti-Humean production must have in mind. For instance, advocates of best-systems accounts maintain that the laws of nature at a given possible world depend on the global structure of the Humean Mosaic at that world (Lewis 1994). This is in turn readily interpreted as a claim about ontological dependence:

(L) The laws of nature at a given world depend for their existence (i.e. their subsistence as real but abstract things) upon the global structure of its Humean Mosaic.

Just as an advocate of Humean Supervenience will claim that there is no possible world containing nothing except a single electron at rest in which the actual laws of nature hold, so too a thermodynamic reductionist can insist that there is no possible world containing nothing except a single electron at rest in which time passes, or in which the laws produce later states of affairs from an initial state. Similarly, a reductionist can insist that there is no possible world in which the positive entropy

4 Note that $\mathrm{P} 3$ is entailed by the conjunction of $\mathrm{P} 3^{*}$ and $\mathrm{P} 2 *$ : (P2*) If $X$ produces $B$ from $A$, then $X, A$, and the signs of objects' velocities in $A$ must all exist before $B$ exists. 
gradient of the universe points in the future-to-past direction. Thus there might no possible world containing 'heat death' before a 'Big Crunch'.

This analogy between laws of nature and the direction of time may seem tenuous. After all, according to best-systems accounts, laws of nature are a- or nontemporal. But if we consider a version of thermodynamic reduction that allows time's arrow to reverse (i.e. 'flip'), then the direction of time seems to be something that exists in time-since it can thus change.

Let me grant this asymmetry: laws of nature are atemporal, whereas the direction of time is temporal. Still, this does not undermine my argument. Why believe that it does?

First, one might insist that only temporal entities can genuinely 'produce' or cause physical states of affairs. In this spirit, then, one might object that the laws of nature, as interpreted by Lewis, do not produce the states of affairs they depend upon -rather, laws so construed just summarize or systematize the global structure of the Humean Mosaic. This first worry is overly broad in scope, however. For instance, it arguably also applies to Maudlin's original view: he characterizes laws of nature as "the patterns that nature respects" $(2007,15)$. So, are these 'patterns' abstract? Are they atemporal? It is not clear what Maudlin himself thinks. But three broad options are salient. First, one could allow that laws of nature are abstract, atemporal entities, while still insisting with Maudlin that the laws produce physical states of affairs. But, beyond just being implausible, the claim that abstract patterns literally 'produce' or 'generate' physical states also directly undermines the central claim underlying this first worry: if atemporal laws can produce physical states, then it is not true that only temporal entities can produce physical states. Second, one could instead simply deny that laws of nature 'produce' physical states. But this is an objection to Maudlin's basic account of production, not an objection to its compatibility with a thermodynamic reduction of time's causal arrow. Third, one could insist that laws of nature are temporal, concrete 'patterns'. But then the claim that the laws of nature at world $w$ depend upon the global structure of the Humean Mosaic at $w$ would be no more or less strange than is the claim that time's arrow at $w$ depends upon the global structure of this same Humean Mosaic.

A second, related worry is that atemporal phenomena cannot depend for their existence upon temporal phenomena because atemporal phenomena do not exist, in 
the first place, insofar as existence is a temporal notion. Hence, one might object, it is not true that Lewisian laws of nature ontologically depend upon any physical states. But this second worry is also unconvincing. If one chooses to reserve the term 'exist' for temporal phenomena, and therefore insists that the laws of nature at world $w$ merely subsist - perhaps at $w$, or perhaps at no world at all, simply in a logical space populated by other abstracta - then so be it. One could still analyze ontological dependence in an expansive sense, as encompassing not just depending-for-existence, but also depending-for-subsistence. In this broader sense, then, it seems fair to say on Lewis's behalf that the laws at $w$ ontologically depend upon the global structure of the Humean Mosaic. There is no reason to think that the distinction between dependingfor-existence and depending-for-subsistence is relevant, in itself, to the plausibility of my argument.

Still, one might object that a given thing cannot both produce and ontologically depend upon another thing: one or the other makes sense, but not both at once. However, there is no clear, non-question-begging motivation for this claim. Ultimately, the plausibility of this P3-style claim is likely grounded in the underlying premises that (i) if a temporal thing, $X$, figures in the production of another thing, $Y$, then $X$ exists before $Y$; (ii) if the direction of time can be thermodynamically reduced, then it is a temporal thing; and (iii) if a temporal thing, $X$, ontologically depends upon another temporal thing, $Y$, then $Y$ exists before $X$ does. But I have argued above that (iii) is far from clearly true. Rather, intuitive appeals to (iii) often simply beg the question against the advocate of reduction-plus-production.

Beyond (iii), another potential reason to insist upon the truth of P3 is the worry that if 'production' and 'ontological dependence' are both species of (or back) explanation, then a given thing both producing and ontologically depending on another thing would lead to explanatory circularity. However, there is arguably no damaging form of explanatory circularity involved in claiming that earlier states of the universe (or objects within these states, or laws of nature) causally explain later states, while also claiming that later states non-causally explain at least some features of earlier states-including the signs of objects' velocities therein. ${ }^{5}$ I have already

5 For a broadly similar distinction between metaphysical and scientific explanation, vis-à-vis defending Humean accounts of laws against worries about explanatory circularity, see Loewer 2012b; Bhogal forthcoming. Cf. Lange 2013, 2018. Contra Lange, see Hicks and van Elswyck 2015; Marshall 2015; Miller 2015. 
sketched the relevant kind of non-causal explanation above, and will further elaborate below.

\section{Beyond a Single, Globally-Consistent Direction of Time}

To this end, it will first prove helpful to consider one further worry about the preceding discussion. Namely, one might object that I often seem to presuppose that the reductionist has a 'rule' that looks at worlds and singles out their past-to-future direction. But then what about reductionists who think that there may be no consistent direction of time, in many worlds-i.e., that some regions of a given world may get one arrow of time, others the opposite?

Here a first point to note is that the version of thermodynamic reduction in which there must be a globally-consistent direction of time is, in some sense, the simplest. Emphasis on this simplest version therefore has the rhetorical advantage of allowing us to focus on the basic issue of the putative tension between thermodynamic reduction and metaphysical production, setting aside secondary complications. Insofar as the reductionist who insists upon a single globally-consistent direction still endorses a genuine version of thermodynamic reduction, my basic point stands: thermodynamic reduction is compatible with a metaphysics of production.

Still, it might be objected, surely this is an inappropriate, ad hoc constraint to impose on the thermodynamic reductionist - who has no internal reason to deny the possibility that the direction of time might 'flip' or reverse within a given world? My response is severalfold:

First, it is not ad hoc in any pejorative sense for a thermodynamic reductionist to insist on a globally-consistent direction of time, insofar as she also believes that there is anti-Humean production or causation, and that it is harder to reconcile an antiHumean metaphysics of production or causation with the possibility of 'flips' in the direction of time within a given world. In this case, the restriction to a single, globallyconsistent direction of time could be a cost that one must bear qua thermodynamic reductionist, in order to realize a (putative) gain qua advocate of metaphysical production. This need not be ad hoc or internally inconsistent. It might just reflect a desire to render a thermodynamic reduction of time's causal arrow consistent with a metaphysics of production, due to the independent appeal of each. But positing a global arrow of time is otherwise certainly odd, for thermodynamic reductionists. 
Second, however, there is arguably no distinctive tension between positing 'flips' or reversals in time's arrow and advocating a metaphysics of production. One apparent problem is how to decide precisely where real flips in the direction of time do or do not occur, in a principled manner. But this is a problem for all reductionists who allow for the possibility that the direction of time can flip within a world. Hence, it is not a distinctive problem for the pairing of metaphysical views that I am arguing is coherent, despite prejudice to the contrary.

Another apparent problem is that it may seem strange to posit causal chains, or more broadly relations of asymmetrical 'productive' dependence, flowing in opposite directions in different regions of spacetime within a single world. But the kind of reversals in time's causal arrow that thermodynamic reductionists may choose to allow for could be constrained so that they do not involve time-reversals between regions of spacetime containing causally-interacting entities. For instance, imagine a spacetime with, not a Past Hypothesis, but a Middle Hypothesis: a very low entropy state obtaining on a spacelike hypersurface in the 'middle' of time, with a positive global entropy gradient in either temporal direction. A thermodynamic reductionist may claim that time's causal arrow flips at this 'Middle State'. If she adds, moreover, that states of affairs on either temporal 'side' of the Middle State are causally produced by phenomena in this Middle State (or by the laws acting on this Middle State, etc.), it is not clear that or how this yields conceptual problems not already faced by any reductionist who posits the same flip.

So, let us set aside this sort of causal-thermodynamic node, where reductionists might locate a flip in the direction of time. What about regions where there is no determinate thermodynamic asymmetry, and hence, for reductionists, $n o$ clear direction of time? Again, it is simply not clear that there are any distinctive problems here for reductionists who also wish to posit a robust metaphysics of production. There might be genuine relations of production in some regions of spacetime in a given world, even if production is absent in other regions. This could simply be the right thing to say. Or, at the very least, it is hard to discount this possibility without begging the question against the advocate of reduction-plus-production.

Likewise, note that production could be a matter of degree. That is, it might be that two states of affairs (etc.) can stand in a relation of robust metaphysical production to one another to a greater or lesser extent. Nothing in the basic idea of 
robust production or anti-Humean causation obviously rules this possibility out. And, again, it may simply be the right thing to say-especially if one independently judges a thermodynamic reduction of time's causal arrow to be well-motivated, or at least seriously pursuit-worthy.

\section{Conclusion: Metaphysical Constraints on Boundary Conditions}

Skeptics may still be left wondering: is it legitimate for a thermodynamic reductionist to simply stipulate that certain apparently-possible initial conditions are, in fact, impossible?

Here we must distinguish the claim that a given state of affairs is physically impossible from the claim that it is metaphysically impossible. For instance, one might claim that it is a law of nature that the universe begins with a low-entropy macrostate (Loewer 2007, 300). This is evidently to claim that certain initial conditions are not physically possible. But one might think that such physically impossible initial conditions are still metaphysically possible.

Above, I suggested a stronger position on behalf of thermodynamic reductionists: the direction of time just is the direction of a thermodynamic asymmetry, such that the only possible worlds with initial states are worlds in which these initial states yield - via causal or productive forces - a thermodynamic asymmetry in the direction of causation or production. If true, this would presumably be a case of a posteriori metaphysical necessity, like the identity of water and $\mathrm{H}_{2} \mathrm{O}$ or down and the direction of a gravitational gradient (compare Sklar 1981). On this view, the point is not that some metaphysically possible initial states are physically impossible, but rather that some metaphysically (and even physically) possible states are metaphysically impossible initial states. Hence thermodynamic reductionists, including those who embrace metaphysical production, can accept that every metaphysically possible initial state is a physically possible initial state, just as antireductionists might claim. Perhaps this is a reason to favor the stronger form of thermodynamic reduction, in which the alignment of the thermodynamic arrow with the cause-to-effect direction is metaphysically necessary, to the weaker version in which this alignment is only physically necessary.

A thermodynamic reduction of time's causal arrow is compatible with a generic anti-Humean metaphysics of production or causation. The reductionist's basic 
claim, that the temporal asymmetry whereby causes typically precede their effects is grounded in an underlying thermodynamic asymmetry, is compatible with the claim that something-whether or not it be the laws of nature - produces later states of affairs from earlier ones, in a manner consistent with anti-Humean notions of production or causation. Arguments to the contrary often beg the question. Indeed, I have worked above to sketch a coherent scheme of reduction-plus-production, combining the intuitive force of both views.

\section{References}

Albert, David Z. 2000. Time and Chance. Harvard University Press.

Albert, David Z. 2015. After Physics. Harvard University Press.

Bhogal, Harjit. Forthcoming. "Nomothetic explanation and Humeanism about laws of nature." Oxford Studies in Metaphysics.

Boltzmann, Ludwig. 1895. "On Certain Questions of the Theory of Gases." Nature 51:41315.

Dowe, Phil. 1992. "Process Causality and Asymmetry." Erkenntnis 37 (2): 179-196.

Earman, John. 1974. "An Attempt to Add a Little Direction to 'The Problem of the Direction of Time'." Philosophy of Science 41 (1): 15-47.

Hicks, Michael Townsen, and Peter van Elswyk. 2015. "Humean laws and circular explanation." Philosophical Studies 172:433-443.

Horwich, Paul. 1987. Asymmetries in Time: Problems in the Philosophy of Science. MIT Press.

Lange, Marc. 2013. "Grounding, scientific explanation, and Humean laws." Philosophical Studies 164:255-261.

Lange, Marc. 2018. "Transitivity, self-explanation, and the explanatory circularity argument against Humean accounts of natural law." Synthese 195:1337-1353.

Lewis, David. 1994. "Humean Supervenience Debugged.” Mind 103:473-490.

Loewer, Barry. 2007. "Counterfactuals and the Second Law." In Causation, Physics, and the Constitution of Reality: Russell's Republic Revisited, ed. Huw Price and Richard Corry, 293-326. Oxford University Press. 
Loewer, Barry. 2012a. “The Emergence of Time's Arrows and Special Science Laws from Physics." Interface Focus 2 (1): 13-19.

Loewer, Barry. 2012b. “Two accounts of Laws and Time.” Philosophical Studies 160:115137.

Marshall, Dan. 2015. "Humean laws and explanation." Philosophical Studies 172:3145-3165.

Maudlin, Tim. 2007. The Metaphysics Within Physics. Oxford University Press.

Miller, Elizabeth. 2015. "Humean scientific explanation." Philosophical Studies 172:13111332.

Price, Huw, and Brad Weslake. 2009. "The time-asymmetry of causation." In The Oxford Handbook of Causation, ed. Helen Beebee, Peter Menzies, and Christopher Hitchcock, 414-43. Oxford University Press.

Reichenbach, Hans. 1956. The Direction of Time, ed. Maria Reichenbach. University of California Press.

Sklar, Lawrence. 1981. "Up and Down, Left and Right, Past and Future." Nô̂s 15 (2): 111129. 